\title{
DNSS2: improved ab initio protein secondary structure prediction using advanced deep learning architectures
}

\author{
Jie $\mathrm{Hou}^{1}$, Zhiye Guo ${ }^{1}$, and Jianlin Cheng ${ }^{1, *}$ \\ ${ }^{1}$ Department of Electrical Engineering and Computer Science, University of Missouri, Columbia, Missouri, \\ 65211, USA.
}

*To whom correspondence should be addressed. Email: chengji@missouri.edu

\begin{abstract}
Motivation: Accurate prediction of protein secondary structure (alpha-helix, beta-strand and coil) is a crucial step for protein inter-residue contact prediction and ab initio tertiary structure prediction. In a previous study, we developed a deep belief network-based protein secondary structure method (DNSS1) and successfully advanced the prediction accuracy beyond $80 \%$. In this work, we developed multiple advanced deep learning architectures (DNSS2) to further improve secondary structure prediction.

Results: The major improvements over the DNSS1 method include (i) designing and integrating six advanced one-dimensional deep convolutional/recurrent/residual/memory/fractal/inception networks to predict secondary structure, and (ii) using more sensitive profile features inferred from Hidden Markov model (HMM) and multiple sequence alignment (MSA). Most of the deep learning architectures are novel for protein secondary structure prediction. DNSS2 was systematically benchmarked on two independent test datasets with eight state-of-art tools and consistently ranked as one of the best methods. Particularly, DNSS2 was tested on the 82 protein targets of 2018 CASP13 experiment and achieved the best Q3 score of $83.74 \%$ and SOV score of $72.46 \%$. DNSS2 is freely available at: https://github.com/multicom-toolbox/DNSS2.
\end{abstract}

\section{Introduction}

Three major types of protein secondary structure are alpha-helix $(\mathrm{H})$, betastrand (E) and coil state (C) (Pauling, et al., 1951), each of which represents the local structure state of an amino acid in a folded polypeptide chain. The predicted information of protein secondary structure is useful for many applications in computational biology, such as protein residueresidue contact prediction (Adhikari, et al., 2017; Michel, et al., 2018; Wang, et al., 2017), protein folding (Hou, et al., 2017; Jones, et al., 1999; Myers and Oas, 2001), ab-initio protein structure modeling (Adhikari and Cheng, 2018; Rohl, et al., 2004; Roy, et al., 2010) and protein model quality assessment (Cao and Cheng, 2016; Uziela, et al., 2016). For instance, secondary structure prediction was widely utilized in the template-based structure modeling through threading or comparative modeling on those proteins that have structurally determined homologs (Roy, et al., 2010; Wang, et al., 2010; Webb and Sali, 2014), and in ab initio modeling for those proteins whose sequences share few sequential similarities with known solved structures (Kryshtafovych, et al., 2017; Ovchinnikov, et al., 2017).

The progress in protein secondary structure prediction over the past few decades can be generally summarized from two aspects: the discovery of novel features that are useful for prediction and the development of effective machine learning algorithms (Rost, 2001; Yang, et al., 2016). The early attempts utilized statistical propensities of single amino acid observed from known structures to identify secondary structures in proteins (Chou and Fasman, 1974). The subsequent improvements came from the inclusion of sequence evolutionary profile features inferred from multiple sequence alignment (MSA) such as position-specific scoring matrices (PSSM) (Altschul, et al., 1997; Dor and Zhou, 2007; Jones, 1999; Magnan and Baldi, 2014; Pollastri and Mclysaght, 2004; Pollastri, et al., 2002). In addition to the PSSM, the Hidden Markov model (HMM) profiles derived from HHblits (Remmert, et al., 2012) was proposed for predicting protein structural properties (Meng, et al., 2018). Atchley's factors were also included in some studies to capture the similarity between the types of amino acids (Atchley, et al., 2005; Spencer, et al., 2015).

Meanwhile, the machine learning algorithms for protein secondary structure prediction also continued to improve. Several early approaches applied shallow neural networks (Holley and Karplus, 1989; Qian and Sejnowski, 1988), information theory and Bayesian analysis (Gibrat, et al., 1987; Schmidler, et al., 2000; Stolorz, et al., 1992) to secondary structure prediction. PSIPRED (Jones, 1999) method proposed a two-stage neural network to predict the secondary structure from the PSI-BLAST sequence profiles. SSpro (Pollastri, et al., 2002) used bi-directional recurrent neural networks to capture the long-range interactions between amino acids. Deep learning techniques recently achieved significant success in secondary structure prediction (Dor and Zhou, 2007; Fang, et al., 2018; Faraggi, et al., 2012; Heffernan, et al., 2017; Spencer, et al., 2015; Wang, et al., 2016). DNSS (Spencer, et al., 2015) applied an ensemble of deep belief networks to predict 3-state secondary structure. SPIDER2 (Heffernan, et al., 2015) employed stacked sparse auto-encoder neural networks to predict the several structural properties iteratively, and this method was further advanced by bidirectional long- and short-term memory (LSTM) neural networks to capture the long-range interactions (Heffernan, et al., 2017). DeepCNF (Wang, et al., 2016) integrated the convolutional neural networks with conditional random-field to learn the complex sequence-structure relationship and interdependence between sequence and secondary structure. Porter 5.0 (Torrisi, et al., 2018) ensembled seven bidirectional recurrent neural networks to improve the protein structure prediction. Assisted with the power of deep learning, the accuracy of 3-state secondary structure prediction has been successfully improved above 84\% (Fang, et al., 2018; Heffernan, et al., 2017; Wang, et al., 2016) on some benchmark datasets. 
bioRxiv preprint doi: https://doi.org/10.1101/639021; this version posted May 17, 2019. The copyright holder for this preprint (which was not certified by peer review) is the author/funder, who has granted bioRxiv a license to display the preprint in perpetuity. It is made available under aCC-BY-NC-ND 4.0 International license.

Jie Hou, et al.

In this work, we developed an improved version of our ab initio secondary structure method using multiple advanced deep learning architectures (DNSS2). Three major improvements have been made over the original DNSS method. Firstly, besides the PSSM profile features and Atchley's factors used in DNSS, we incorporated several novel features such as the emission and transition probabilities derived from Hidden Markov model (HMM) profile (Remmert, et al., 2012), and profile probabilities inferred from multiple sequence alignment (MSA) (Magnan and Baldi, 2014). All the three new features represent the evolutionary conservation information for amino acids in sequence. Secondly, we designed and integrated six types of advanced one-dimensional deep networks for protein secondary structure prediction, including traditional convolutional neural network (CNN) (Krizhevsky, et al., 2012), recurrent convolutional neural network (RCNN) (Liang and Hu, 2015), residual neural network (ResNet) (He, et al., 2016), convolutional residual memory networks (CRMN) (Moniz and Pal, 2016), fractal networks (Larsson, et al., 2016), and Inception network (Szegedy, et al., 2015). The ensemble of six networks from DNSS2 significantly improved the secondary structure prediction. Finally, DNSS2 was trained on a large dataset, including 4,872 non-redundant protein structures with less than $25 \%$ pairwise sequence identity and $2.5 \AA$ resolution. Our method was extensively tested on the independent dataset and the latest CASP13 dataset with other state-of-art methods and delivered the state-of-the-art performance.

\section{Materials and Methods}

\subsection{Experimental design}

In this work, the main objective was to improve the secondary structure prediction by developing more advanced deep learning architectures and introducing more useful features. In the process, we have developed a systematic framework to effectively build deep learning architectures and obtain features to improve secondary structure prediction. Figure 1 provides an overview of our experimental design. Figure 1(A) lists the six major steps of designing, training and testing deep learning architectures. Figure 1(B) illustrates the process of creating training and validation datasets. The key analysis is to design appropriate architectures and investigate if they can improve prediction accuracy. Six different deep neural network architectures were evaluated in the study, including convolutional neural network (CNN) (Krizhevsky, et al., 2012), recurrent convolutional neural network (RCNN) (Liang and Hu, 2015), ResNet (He, et al., 2016), convolutional recurrent memory network (CRMN) (Moniz and Pal, 2016), FractalNet (Larsson, et al., 2016), and Inception network (Szegedy, et al., 2015). Most of these architectures were applied to secondary structure prediction for the first time. The detailed description of each network is included in Section 2.4. To ensure a fair comparison, each network was optimized using the original feature profiles of training proteins and evaluated on the same validation set of DNSS1. The network that achieved the best Q3 accuracy was selected to explore the feature space on the profiles derived from multiple sequence alignments (MSA) generated by PSIBLAST (Altschul, et al., 1997) and HHblits (Remmert, et al., 2012), Atchley factors, and emission/transition probabilities inferred from the Hidden Markov model (HMM) profile. The optimal feature set was determined according to the highest Q3 accuracy on the validation datasets. The networks were then re-trained using the optimal input profiles to obtain the best models.

Since combining predictors generally improved the prediction accuracy, the different combinations of networks were also evaluated. Finally, after the optimal sets of deep learning architectures and feature profiles were determined, all networks were re-trained on the large dataset that was manually curated including the non-redundant proteins whose structures have been released publicly before 2018 . The final networks were used to predict the secondary structure for the test proteins. The probabilities of the three states (i.e. helix, sheet, and coil) for each residue predicted by six networks were averaged to make the final secondary structure prediction.

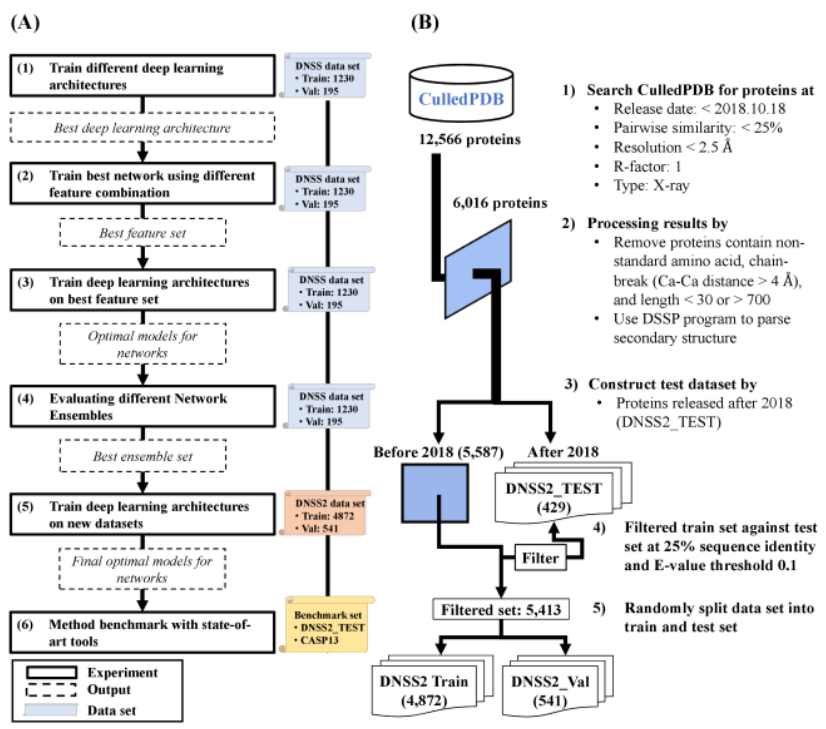

Fig. 1. Overview of the experimental workflow for improving secondary structure prediction. (A) Six principal steps are conducted to construct and train deep networks. The solid box represents an analysis step. The dashed box represents the output from the previous step. The scroll represents the dataset used in each step. (B) Dataset generation and filtering process.

Our method was then benchmarked with other state-of-art methods on the two independent test datasets.

\subsection{Datasets and evaluation metric}

As described in section 2.1, two training datasets were used in our experiment. In the first stage, the original DNSS dataset (Spencer, et al., 2015) that included 1,230 training proteins and 195 validation proteins was utilized to investigate whether the deep learning architectures and novel features can boost the prediction accuracy.

To utilize more data available since DNSS1 was published, a new, larger training set of DNSS2 was constructed from CullPDB (Wang and Dunbrack Jr, 2003) curated on 18 October 2018 (Figure 1(B)). The dataset consists of 12,566 proteins that share less than $25 \%$ sequence identity with $2.5 \AA$ resolution cutoff and R-factor cutoff 1 . The structures of all the proteins were determined by X-ray crystallography. The dataset was then filtered by removing proteins with non-standard amino acids, chain-break (i.e. distance of adjacent $\mathrm{Ca}-\mathrm{Ca}$ atoms is larger than $4 \AA$ ), and sequence length shorter than 30 or longer than 700 amino acids. Considering all external methods benchmarked in this work were developed prior to year 2018, the proteins that were released after Jan $1^{\text {st }}, 2018$ were extracted as independent test set (DNSS2_TEST). The resulting set of proteins was further filtered against DNSS2_TEST set using CD-HIT suite ( $\mathrm{Li}$ and Godzik, 2006) with criteria of $25 \%$ sequence identity cutoff and e-value threshold 0.1. Finally, 5,413 proteins released prior to Jan $1^{\text {st }}, 2018$ were obtained as our training set, in which 4,872 proteins were used for network training (DNSS2_TRAIN) and 547 proteins were used for model selection (DNSS2_VAL). In addition, the proteins of the CASP13 (2018) experiment were collected and the ones with at least $25 \%$ sequence identity with 
bioRxiv preprint doi: https://doi.org/10.1101/639021; this version posted May 17, 2019. The copyright holder for this preprint (which was not certified by peer review) is the author/funder, who has granted bioRxiv a license to display the preprint in perpetuity. It is made available under aCC-BY-NC-ND 4.0 International license.

DNSS2: improved ab initio protein secondary structure prediction using advanced deep learning architectures

training proteins were removed, which results in a set of 82 test proteins. The proteins were also classified into template-based (TBM) and freemodeling (FM) targets based on the official CASP definition (CASP 13, 2018, http://www.predictioncenter.org/casp13/index.cgi). In summary, the final test set contain 429 proteins from DNSS2_TEST and 82 proteins from CASP13.

We evaluated our secondary structure prediction based on two primary metrics: Q3 accuracy and Segment Overlap measure (SOV). Q3 score represents the percent of correctly predicted secondary structure states in a protein. SOV score measures the similarity between the predicted segments of continuous structure states and those in the experimental structure (Spencer, et al., 2015; Zemla, et al., 1999). The Q3 and SOV scores are complementary with each other for secondary structure evaluation. All training and testing proteins' structure files were parsed by DSSP program (Kabsch and Sander, 1983) to obtain the real secondary structure classification for each amino acid for training and evaluation.

\subsection{Input features}

The profile of each amino acid is represented by 21 numbers from PSIBLAST-based position specific scoring matrix (PSSM), 20 emission probabilities and 7 transition probabilities extracted from Hidden Markov Model (HMM) profile, 20 probabilities of standard amino acid calculated from the multiple sequence alignment (MSA) and 5 numbers derived from Atchley's factor. These features (73 numbers in total) represent the evolutionary conservation and physicochemical properties for residues in a protein sequence.

PSI-BLAST was run to generate multiple sequence alignment and PSSM profile through searching a sequence against filtered UniProt sequence database at $90 \%$ sequence identity (UniRef90) (Consortium, 2014) with three iterations and an e-value cutoff 0.001 ('-evalue .001 -inclusion_ethresh .002'). Less stringent threshold was used ('-evalue 10 -inclusion_ethresh 10') in case some proteins did not have homologous sequences returned. In a PSSM profile, each position is represented by 20 numbers related to the probabilities for 20 standard amino acids appearing at the position in the multiple sequence alignment. In addition, the sequence information in the second to the last column in PSI-BLAST profile is given for each residue.

HMM profile was generated by running three iteration of 'HHblits' against the uniclust30 database (version: October 2017) (Mirdita, et al., 2016). Two types of probabilities were associated with each residue in a HMM profile: emission probability and transition probability. Emission probability represents the probability of a given amino acid occurring at the position in the multiple sequence alignment. The transition probability represents the probability transiting from an alignment state (i.e. match, insertion, and deletion) to another. Similar to PSSM, the emission frequencies of the 20 standard amino acid for each residue were reported in the HMM profile, and the probabilities were calculated according to formula:

$$
p_{i k}=2^{\left(-\frac{{ }^{F r e q}}{1000}\right)}
$$

where $i$ is the $i$-th residue in sequence and $k$ is the $k$-th standard amino acid. And the probability is set to 0 if the frequency is denoted as ' *'. The transition probabilities for each amino acid were also derived in the same fashion. In total, 20 emission probabilities and 7 transition probabilities for each amino acid were collected to represent the residue conservation inferred from HMM.

Since HHblits was more sensitive to identify distant homologous sequences than PSI-BLAST, the probability matrix of amino acids was also calculated from the multiple sequence alignment (MSA) generated by HHblits. The conversion from MSA to a probability matrix follows the same calculation as SSpro (Magnan and Baldi, 2014).

\subsection{Deep learning architectures}

A widely used deep learning architecture in bioinformatics is deep convolutional neural networks (CNN). Convolutional neural networks have some distinctive advantages over the traditional neural networks for the bioinformatics problems in several ways: (1) it can learn informative representation directly from sequence features without requiring segmentation (e.g. sliding window) or dimension reduction (e.g. principle component analysis) techniques; (2) the convolutional network can learn both local and global features to discover complex patterns; and (3) the architecture is independent of input size (i.e. length or volume). In this work, we design a standard CNN and five advanced deep learning architectures based on both convolutional and other useful operations as in Figure 2.

Figure 2(A) illustrates our standard convolutional neural network $(\mathrm{CNN})$ for secondary structure prediction, consisting of a sequence of convolutional blocks, each of which contains a convolutional layer, a batch-
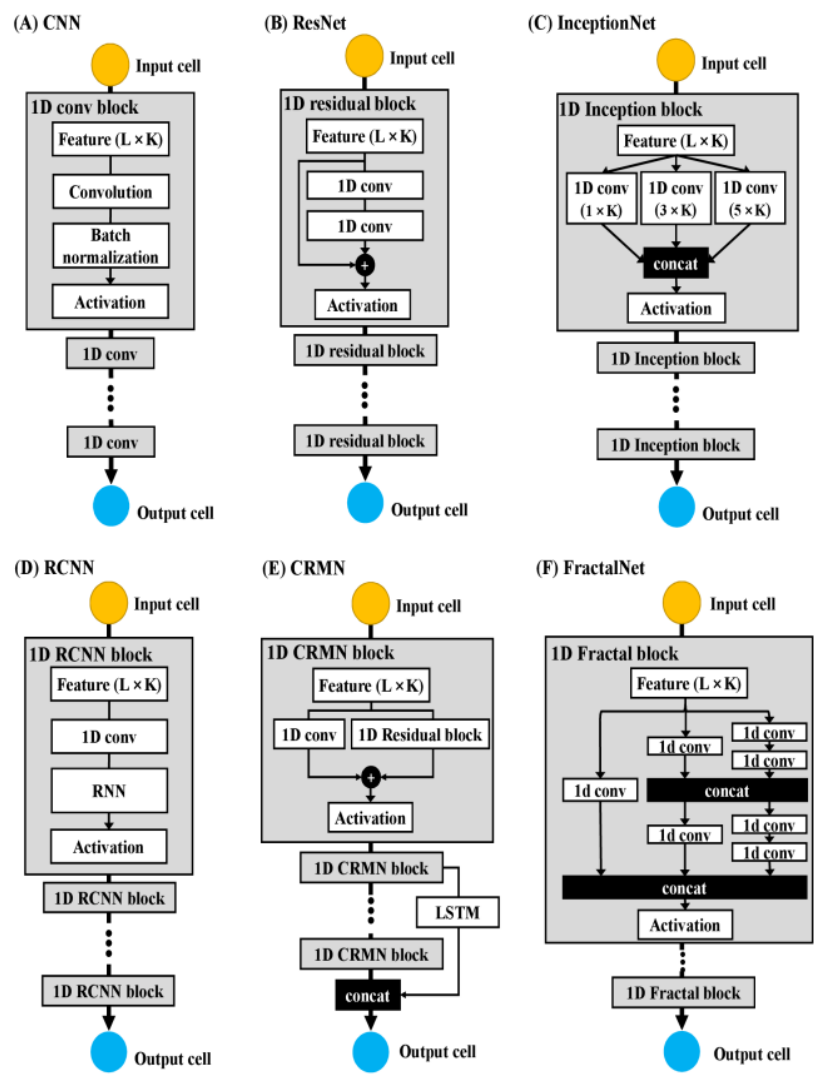

Fig. 2. Six deep learning architectures: (A) CNN, (B) ResNet, (C) InceptionNet, (D) RCNN, (E) CRNN, (F) FractalNet) for secondary structure prediction. L: sequence length; K: number of features per position.

normalization layer, and an activation layer. The original input is a $\mathrm{L} \times \mathrm{K}$ vector $(X)$, where $\mathrm{L}$ is sequence length and $\mathrm{K}$ is the number of features per residue position in the sequence. For each convolution block, the feature maps are obtained after the convolution operation is applied by multiplying the weight matrices (called filters, $W$ ) with a window of local features on the previous input layer and adding bias vectors $(b)$ according to the formula: $X^{l+1}=W^{l+1} * X^{l}+b^{l+1}$, where $l$ is the layer number. The batch normalization layer is added to obtain a Gaussian normalization of convolved features coming out of each convolutional layer. Then an activation function such as rectified linear function (i.e. ReLU) is applied to 
Jie Hou, et al.

extract non-linear patterns of the normalized hidden features. To avoid overfitting, regularization approaches such as dropout (Srivastava, et al., 2014) can be applied in the hidden layers. The final output node (also a filter) in the output cell uses the softmax function to classify the input of each residue position from its previous layer into one of three secondary structure states. The output is a $\mathrm{L} \times 3$ vector, holding the predicted probability of three secondary structure states for each of $\mathrm{L}$ positions in a sequence. The final optimal CNN architecture includes 6 convolutional blocks, in which the filter size (window size) for each convolutional layer is 6 , and the number of filters (feature maps) in each convolution layer is 40.

The residual network (ResNet) was designed to make traditional convolutional neural network deeper without gradient vanishing. The architecture constructs many residual blocks and stacked up them to form a deeper network, as shown in Figure 2 (B). In each residual block, the input $X^{l}$ is fed into a few convolutional layers to obtain the non-linear transformation output $G\left(X^{l+1}\right)$. In order to make the network deeper, an extra skip connection (i.e. short-cut) is added to copy the input $X^{l}$ to the output of non-linear transformation layer, where $X^{(l+1) *}$ can be represented as $X^{(l+1) *}=X^{l}+G\left(X^{l+1}\right)$ before applying another ReLU non-linearity. This process makes neural network deeper by adding shortcuts to facilitate gradient back-propagation during training and achieve better performance. The residual blocks with different configuration can be stacked to achieve higher accuracy. For instance, the final best architecture in DNSS2 is made up of 13 residual blocks, each of which includes 3 convolutional layers with filter size 1, 3, 1 respectively. The first three residual blocks used 37 filters to learn features, while the middle four blocks used 74 filters for each convolution layer, and the last six residual blocks used 148 filters. In total, 39 convolutional layers are included in the final residual network. In the network, the dropout and batch normalization were also added to prevent network from overfitting.

Inception network is an advanced architecture for building deeper networks by repeating a bunch of inception modules, as shown in Figure 2(c). Instead of trying to determine the best values for certain hyper-parameters (i.e. number of filter size, number of layers, inclusion of pooling layer), inception network proposes to concatenate outputs of hidden layers with different configuration through an inception module and trains the network to learn patterns from the combination of diverse hyper-parameters. Despite its high computation cost, inception network has performed remarkably well in many applications (Fang, et al., 2018; Szegedy, et al., 2015). For secondary structure prediction, a combination of three filter sizes $1 \times \mathrm{K}, 3 \times \mathrm{K}$ and $5 \times \mathrm{K}$ was applied to convolve feature input, where $\mathrm{K}$ is the number of original input features for each residue position. The concatenation of the convolution outputs is fed into an activation layer for non-linear activation calculation. This kind of inception module is repeated to make a deeper network. After the parameter tuning, the optimal inception network is comprised of three inception blocks with 24 convolution layers included.

In addition, we designed three more deep learning architectures: recurrent convolutional neural network (RCNN) (Liang and Hu, 2015), convolutional residual memory networks (CRMN) (Moniz and Pal, 2016), and fractal network for secondary structure prediction. The recurrent convolutional neural network (RCNN) was designed to model sequential dependency hidden inside the sequential features (Figure 2(D)), It firstly extracts the higher-level feature maps by a convolution block, and then uses a recurrent neural network (i.e. bi-directional Long-Short-Term Memory (LSTM) network) for modeling the inter-dependence among the convolved features. Such a recurrent convolutional block with 4 convolutional layers included is repeated 5 times to build a deep recurrent convolutional neural network for secondary structure prediction in this work. The
CRMN network augmented the architectures by integrating convolutional residual networks with LSTM (Figure 2(E)) (e.g., 2 residual blocks and 2 LSTM in the network). Both methods advanced the convolutional neural network by introducing the memory mechanisms of recurrent neural network (RNN). Moreover, inspired by ResNet and Inception Network, we built a Fractal network stacking up different number of convolution blocks in both parallel and hierarchical fashion by adding several shortcut paths to connect lower-level layers and higher-level layers, as shown in Figure 2(F). After tuning, the fractal network was assembled with 16 convolution layers for one fractal block.

\subsection{Training and evaluation procedure}

Deeper networks with complex architectures are generally difficult to train effectively due to the high-dimensional hyper-parameter space. To obtain good performance on specific feature sets within a reasonable amount of time for each deep network, we developed an efficient heuristic random sampling approach for model hyperparameter optimization. Specifically, based on the several trials on network training, we first determined heuristically a reasonable range for each type of the network hyperparameters, including the number of filters from 20 to 50 , the number of convolution blocks from 3 to 7 , and the filter size from 3 to 7 . For each subsequent trial, the values of hyper-parameters were randomly sampled from their specified range and the Q3 accuracy of the network on the validation dataset under the specific parameter combination was assessed. For each deep network, the best parameter set was determined after 100 trials were evaluated. We found that using the random sampling technique was able to generate better models in most cases and was also more efficient than the traditional grid search or greedy search.

The performance of different deep architectures and different feature profiles on the secondary structure prediction were rigorously examined using the training and validation set from original DNSS method. After the parameters and input features were determined, we trained each deep network on the latest curated dataset (DNSS2_TRAIN) and selected best models using the Q3 accuracy on the independent validation dataset (DNSS2_VAL). We used the Keras library (http://keras.io/) along with Tensorflow as a backend to train all networks.

The performance of DNSS2 was evaluated on the two independent datasets and compared with a variety of the state-of-art secondary structure prediction tools, including SSpro5.2 (Magnan and Baldi, 2014), PSSpred (Yan, et al., 2013), MUFOLD-SS (Fang, et al., 2018), DeepCNF (Wang, et al., 2016), PSIPRED (McGuffin, et al., 2000), SPIDER3 (Heffernan, et al., 2017), Porter 5 (Torrisi, et al., 2018) and our previous method DNSS1 (Spencer, et al., 2015). All the methods were assessed according to the Q3 and SOV scores on each dataset.

\section{Results}

\subsection{Benchmarking different deep architectures of DNSS2 with DNSS1}

The first evaluation was to investigate whether the new deep architectures networks (DNSS2) outperform the deep belief network (DNSS1) for the secondary structure prediction. In order to fairly compare them, we trained and validated the six deep networks on the original input features of the same 1,230 training and 195 validation proteins used to train and test DNSS1. Table 1 compares the Q3 and Sov scores of DNSS1 and DNSS2 architectures on the validation set. The results show that five out of six new advanced deep networks (RCNN, ResNet, CRMN, FractalNet, and InceptionNet) except the standard CNN network obtain higher Q3 scores than the deep belief network that used in DNSS1. InceptionNet worked best among individual deep architectures. The ensemble of the six deep 
bioRxiv preprint doi: https://doi.org/10.1101/639021; this version posted May 17, 2019. The copyright holder for this preprint (which was not certified by peer review) is the author/funder, who has granted bioRxiv a license to display the preprint in perpetuity. It is made available under aCC-BY-NC-ND 4.0 International license.

DNSS2: improved ab initio protein secondary structure prediction using advanced deep learning architectures

architectures (DNSS2) achieved the highest Q3 score of $83.04 \%$, better than all the six individual deep architectures and 79.1\% Q3 score of DNSS1.

\begin{tabular}{lll}
\hline Method & Q3(\%) & Sov(\%) \\
\hline DNSS1 & 79.1 & 72.38 \\
DNSS2_CNN & 77.86 & 68.42 \\
DNSS2_RCNN & 79.87 & 72.34 \\
DNSS2_ResNet & 79.61 & 69.94 \\
DNSS2_CRMN & 79.32 & 69.21 \\
DNSS2_FractalNet & 79.85 & 72.82 \\
DNSS2_InceptionNet & 80.68 & 72.74 \\
DNSS2 & 83.04 & 72.74 \\
\hline
\end{tabular}

Table 1. Performance of the six different deep architectures and their ensemble on the DNSS1 validation dataset. DNSS2 represents the ensemble of six deep architectures (CNN, RCNN, ResNet, CRMN, FractalNet and InceptionNet).

\subsection{Impact of different input features}

After the best deep learning architecture (i.e. InceptionNet) was determined, it was utilized to examine the impact of the different input features including PSSM, Atchley factor (FAC), Emission probabilities (Em), Transition probabilities ( $\mathrm{Tr}$ ), and amino acids probabilities from HHblits alignments (HHblitsMSA). In this analysis, the protein sequence databases required for alignment generation were updated to latest and all the input features for DNSS1 datasets were regenerated. Specifically, the Uniref 90 database that was released at October 2018 was used to generate PSSM profiles by PSI-BLAST, and the latest version of Uniclust30 database (October 2017) was used to generate HMM profiles by HHblits. The Inception network was then trained on the 1,230 proteins using the combination of five kinds of features. We tested six feature combinations shown in Table 2. Hyper-parameter optimization was applied to obtain the best model on each feature combination. Table 2 shows the performance of different input feature combinations with the inception network on the validation dataset of 195 proteins. Adding the emission profile inferred from HMM model on top of PSSM and Atchley factor features increased the Q3 score from $79.81 \%$ to $82.31 \%$. Integrating all the five kinds of features will yield the highest Q3 score (i.e. 82.72\%) and Sov score $(75.89 \%)$.

The performance of the six deep architectures and their ensemble on the latest features (the combination of all five kinds of features) of the DNSS1 validation dataset was also reported in Table 3. All six architectures were re-trained on the 1,230 proteins and evaluated on the validation dataset. Compared to the results in Table 1, the prediction accuracy of all the networks on the validation set was improved. The Q3 and SOV scores of the ensemble (DNSS2) were increased to $83.84 \%$ and $75.5 \%$, respectively. The results indicate that the update of the protein sequence databases helps improve prediction accuracy.

\begin{tabular}{llll}
\hline Rank & Feature Name & Q3(\%) & SOV $(\%)$ \\
\hline 1 & PSSM + FAC + Em + Tr + 82.72 & 75.89 \\
& HHblitsMSA & & \\
2 & PSSM + FAC + Em + Tr & 82.36 & 76.03 \\
3 & PSSM + FAC + Em & 82.31 & 74.15 \\
4 & PSSM + FAC + HHblitMSA & 81.98 & 74.67 \\
5 & PSSM + FAC + Tr & 80.13 & 71.61 \\
6 & PSSM + FAC & 79.81 & 71.43 \\
\hline
\end{tabular}

Table 2. Performance of different input feature combinations on the validation dataset of 195 proteins. PSSM, FAC, Em, Tr, HHblitsMSA denote five kinds of features: PSSM, Atchley factor, Emission probabilities, Transition probabilities, amino acid probabilities from HHblits alignments.

\begin{tabular}{lll}
\hline Method & Q3(\%) & Sov(\%) \\
\hline DNSS2_CNN & 80.29 & 72.1 \\
DNSS2_RCNN & 81.83 & 73.97 \\
DNSS2_ResNet & 81.53 & 73.71 \\
DNSS2_CRMN & 81.91 & 73.37 \\
DNSS2_FractalNet & 82.02 & 73.8 \\
DNSS2_InceptionNet & 82.74 & 75.3 \\
DNSS2 & 83.84 & 75.5 \\
\hline
\end{tabular}

Table 3. Performance of the six different deep learning architectures (CNN, RCNN, ResNet, CRMN, FractalNet, and InceptionNet) and their ensemble (DNSS2) on DNSS1 validation dataset and the updated protein sequence database.

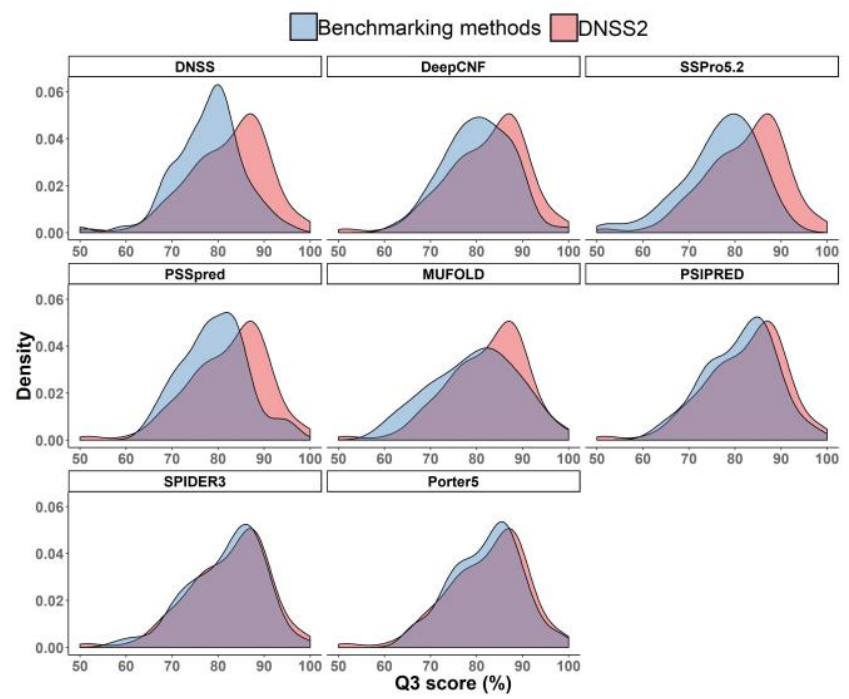

Fig. 3. Comparison of the distribution of Q3 scores of eight existing methods and that of DNSS2 on all CASP13 targets.

\subsection{Comparison of DNSS2 with eight state-of-the-art tools on two independent test datasets}

DNSS2 was compared with eight state-of-art methods including SSPro5.2, DNSS1, PSSpred, MUFOLD-SS, DeepCNF, PSIPRED, SPIDER3, and Porter 5 on the DNSS2_TEST dataset. The test dataset contains nonredundant proteins released after Jan $1^{\text {st }}$, 2018. All the tools were downloaded and configured based on their instructions. The sequence databases that the tools require were updated to the latest version.

The Q3 score of each tool on the test dataset was reported in Table 4. In general, DNSS2 is comparable to the two predictors (Porter 5 and SPIDER3) on this dataset and outperforms the other six methods. Specifically, DNSS2 achieved a Q3 accuracy of $85.02 \%$ and SOV accuracy of $76.01 \%$ on the DNSS2_TEST dataset, which was significantly better than DNSS 1.0 on the DNSS2_test dataset with p-value equal to 2.2E-16.

In addition to the DNSS2_test dataset, we also compared these methods on the 82 protein targets of 2018 CASP13 experiment, which share less 
bioRxiv preprint doi: https://doi.org/10.1101/639021; this version posted May 17, 2019. The copyright holder for this preprint (which was not certified by peer review) is the author/funder, who has granted bioRxiv a license to display the preprint in perpetuity. It is made available under aCC-BY-NC-ND 4.0 International license.

Jie Hou, et al.

than $25 \%$ sequence identity with the training proteins of DNSS2. Both template-based (TBM) and free-modeling (FM) protein targets were used to evaluate the methods and the results are summarized in the Table $\mathbf{5}$. Consistent with the performance on the DNSS2_test dataset shown in Table 4, DNSS2, SPIDER3 and Porter 5 performed best, while DNSS2 achieved slightly better performance than SPIDER3 and Porter 5. Figure 3 plots the distribution of the Q3 scores for all CASP13 targets obtained by DNSS2 and the other eight methods. In general, the distribution of DNSS2 consistently shifts to higher Q3 score compared with other methods, even though the distribution of DNSS2 largely overlaps with that of SPIDER3 and Porter 5.

Table 6 summarized the confusion matrix of predictions of three kinds of secondary structures (helix, sheet, coil) by DNSS2 on the CASP13 dataset. DNSS2 yields the highest accuracy for helical prediction (87.91\%), followed by the coil prediction $(80.21 \%)$ and the sheet prediction (76.45\%). The prediction errors between helix, sheet, and coil was also reported. The error rate of misclassifying helix as sheet is the lowest $(0.57 \%)$ and sheet as coil is the highest $(22.46 \%)$.

\begin{tabular}{lll}
\hline Method & Q3 $(\boldsymbol{\%})$ & SOV $(\boldsymbol{\%})$ \\
\hline SSPro5.2 & 79.26 & 70.78 \\
PSSpred & 81.86 & 71.65 \\
MUFOLD & 81.85 & 73.56 \\
DeepCNF & 82.85 & 70.57 \\
PSIPRED & 83.94 & 74.49 \\
SPIDER3 & 85.34 & 77.61 \\
Porter 5 & 85.07 & 76.79 \\
DNSS1 & 80.14 & 73.63 \\
DNSS2 & 85.02 & 76.01 \\
\hline
\end{tabular}

Table 4. Q3 scores of 9 secondary structure prediction methods on DNSS2_test dataset. Three methods (SPIDER3, Porter5, DNSS2) have Q3 score higher than $85 \%$.

\section{Conclusion}

In this work, we developed several advanced deep learning architectures and their ensemble to improve secondary structure prediction. We investigated six advanced deep learning architectures and five kinds of input

\section{Reference}

Adhikari, B. and Cheng, J. CONFOLD2: improved contact-driven ab initio protein structure modeling. BMC bioinformatics 2018;19(1):22.

Adhikari, B., Hou, J. and Cheng, J. DNCON2: Improved protein contact prediction using two-level deep convolutional neural networks. Bioinformatics 2017;34(9):1466-1472.

Altschul, S.F., et al. Gapped BLAST and PSI-BLAST: a new generation of protein database search programs. Nucleic acids research 1997;25(17):3389-3402.

Atchley, W.R., et al. Solving the protein sequence metric problem. Proceedings of the National Academy of Sciences 2005;102(18):6395-6400.

Cao, R. and Cheng, J. Integrated protein function prediction by mining function associations, sequences, and protein-protein and gene-gene interaction networks. Methods 2016;93:84-91.

Chou, P.Y. and Fasman, G.D. Prediction of protein conformation. Biochemistry 1974;13(2):222-245 features on secondary structure prediction. Several deep learning architectures such as inception network, fractal network, and recurrent convolutional memory network are novel for protein secondary structure prediction and performed better than the deep belief network. The performance of the deep learning method is comparable to or better than seven external state-of-the-art methods on the two independent test datasets. Our experiment also demonstrated that emission/transition probabilities extracted from hidden Markov model profiles are useful for secondary structure prediction.

\begin{tabular}{lllllll}
\hline & All & & TBM & FM \\
\hline Method & Q3 & SOV & Q3 & SOV & Q3 & SOV \\
& $(\%)$ & $(\%)$ & $(\%)$ & $(\%)$ & $(\%)$ & $(\%)$ \\
\hline SSPro5.2 & 76.73 & 69.94 & 78.16 & 71.32 & 76.12 & 70.88 \\
\hline PSSpred & 78.8 & 67.85 & 81.32 & 72.11 & 76.99 & 64.55 \\
\hline MUFOLD & 79.58 & 71.74 & 79.71 & 74.13 & 79.8 & 70.79 \\
\hline DeepCNF & 80.24 & 69.5 & 82.34 & 73.68 & 78.36 & 65.55 \\
\hline PSIPRED & 80.7 & 72 & 83.67 & 76.72 & 78.41 & 68.14 \\
\hline SPIDER3 & 81.73 & 74.39 & 84.84 & 78.31 & 78.89 & 71.1 \\
\hline Porter5 & 82.07 & 74.61 & 84.79 & 78.98 & 79.42 & 70.3 \\
\hline DNSS1 & 77.06 & 70.40 & 79.48 & 73.58 & 75.46 & 68.79 \\
\hline DNSS2 & 82.2 & 73.03 & 85.37 & 76.98 & 79.82 & 70.56 \\
\hline
\end{tabular}

Table 5. Comparison of methods on the CASP13 dataset in terms of all CASP13 targets, template-based targets, and template-free targets.

\begin{tabular}{llll}
\hline & C pred & E pred & H pred \\
\hline Coil (C) & $80.21 \%$ & $9.51 \%$ & $10.28 \%$ \\
Sheet (E) & $22.46 \%$ & $76.45 \%$ & $1.10 \%$ \\
Helix (H) & $11.52 \%$ & $0.57 \%$ & $87.91 \%$ \\
\hline
\end{tabular}

Table 6. Confusion matrix of helix, sheet and coil predicted by DNSS2 on CASP13 dataset.

\section{Funding}

This work has been supported by an NIH grant (R01GM093123) and two NSF grants (DBI1759934, IIS1763246) to JC.

Conflict of Interest: none declared.

Consortium, U. UniProt: a hub for protein information. Nucleic acids research 2014;43(D1):D204-D212.

Dor, O. and Zhou, Y. Achieving 80\% ten - fold cross - validated accuracy for secondary structure prediction by large - scale training. Proteins: Structure, Function, and Bioinformatics 2007;66(4):838-845.

Fang, C., Shang, Y. and Xu, D. MUFOLD - SS: New deep inception - inside inception networks for protein secondary structure prediction. Proteins: Structure, Function, and Bioinformatics 2018;86(5):592-598.

Faraggi, E., et al. SPINE X: improving protein secondary structure prediction by multistep learning coupled with prediction of solvent accessible surface area and backbone torsion angles. Journal of computational chemistry 2012;33(3):259-267. Gibrat, J.-F., Garnier, J. and Robson, B. Further developments of protein secondary structure prediction using information theory: new parameters and consideration of residue pairs. Journal of molecular biology 1987;198(3):425-443. 
bioRxiv preprint doi: https://doi.org/10.1101/639021; this version posted May 17, 2019. The copyright holder for this preprint (which was not certified by peer review) is the author/funder, who has granted bioRxiv a license to display the preprint in perpetuity. It is made available under aCC-BY-NC-ND 4.0 International license.

DNSS2: improved ab initio protein secondary structure prediction using advanced deep learning architectures

He, K., et al. Deep residual learning for image recognition. In, Proceedings of the IEEE conference on computer vision and pattern recognition. 2016. p. 770-778.

Heffernan, R., et al. Improving prediction of secondary structure, local backbone angles, and solvent accessible surface area of proteins by iterative deep learning. Scientific reports 2015;5:11476.

Heffernan, R., et al. Capturing Non-Local Interactions by Long Short Term Memory Bidirectional Recurrent Neural Networks for Improving Prediction of Protein Secondary Structure, Backbone Angles, Contact Numbers, and Solvent Accessibility. Bioinformatics 2017:btx218.

Holley, L.H. and Karplus, M. Protein secondary structure prediction with a neural network. Proceedings of the National Academy of Sciences 1989;86(1):152-156.

Hou, J., Adhikari, B. and Cheng, J. DeepSF: deep convolutional neural network for mapping protein sequences to folds. arXiv preprint arXiv:1706.01010 2017.

Jones, D.T. Protein secondary structure prediction based on position-specific scoring matrices. Journal of molecular biology 1999;292(2):195-202.

Jones, D.T., et al. Successful recognition of protein folds using threading methods biased by sequence similarity and predicted secondary structure. Proteins: Structure, Function, and Bioinformatics 1999;37(S3):104-111.

Kabsch, W. and Sander, C. Dictionary of protein secondary structure: pattern recognition of hydrogen - bonded and geometrical features. Biopolymers: Original Research on Biomolecules 1983;22(12):2577-2637.

Krizhevsky, A., Sutskever, I. and Hinton, G.E. Imagenet classification with deep convolutional neural networks. In, Advances in neural information processing systems. 2012. p. 1097-1105.

Kryshtafovych, A., et al. Evaluation of the template - based modeling in CASP12. Proteins: Structure, Function, and Bioinformatics 2017.

Larsson, G., Maire, M. and Shakhnarovich, G. Fractalnet: Ultra-deep neural networks without residuals. arXiv preprint arXiv:1605.07648 2016.

Li, W. and Godzik, A. Cd-hit: a fast program for clustering and comparing large sets of protein or nucleotide sequences. Bioinformatics 2006;22(13):1658-1659.

Liang, M. and $\mathrm{Hu}, \mathrm{X}$. Recurrent convolutional neural network for object recognition. In, Proceedings of the IEEE Conference on Computer Vision and Pattern Recognition. 2015. p. 3367-3375.

Magnan, C.N. and Baldi, P. SSpro/ACCpro 5: almost perfect prediction of protein secondary structure and relative solvent accessibility using profiles, machine learning and structural similarity. Bioinformatics 2014;30(18):2592-2597.

McGuffin, L.J., Bryson, K. and Jones, D.T. The PSIPRED protein structure prediction server. Bioinformatics 2000;16(4):404-405.

Meng, Q., et al. CoABind: a novel algorithm for Coenzyme A (CoA)-and CoA derivatives-binding residues prediction. Bioinformatics 2018;1:7.

Michel, M., Hurtado, D.M. and Elofsson, A. PconsC4: fast, accurate, and hassle-free contact predictions. Bioinformatics 2018:bty1036-bty1036.

Mirdita, M., et al. Uniclust databases of clustered and deeply annotated protein sequences and alignments. Nucleic acids research 2016;45(D1):D170-D176.

Moniz, J. and Pal, C. Convolutional residual memory networks. arXiv preprint arXiv:1606.05262 2016.

Myers, J.K. and Oas, T.G. Preorganized secondary structure as an important determinant of fast protein folding. Nature Structural \& Molecular Biology 2001;8(6):552-558.

Ovchinnikov, S., et al. Protein structure prediction using Rosetta in CASP12. Proteins: Structure, Function, and Bioinformatics 2017.

Pauling, L., Corey, R.B. and Branson, H.R. The structure of proteins: two hydrogenbonded helical configurations of the polypeptide chain. Proceedings of the National Academy of Sciences 1951;37(4):205-211.
Pollastri, G. and Mclysaght, A. Porter: a new, accurate server for protein secondary structure prediction. Bioinformatics 2004;21(8):1719-1720.

Pollastri, G., et al. Improving the prediction of protein secondary structure in three and eight classes using recurrent neural networks and profiles. Proteins: Structure, Function, and Bioinformatics 2002;47(2):228-235.

Qian, N. and Sejnowski, T.J. Predicting the secondary structure of globular proteins using neural network models. Journal of molecular biology 1988;202(4):865-884.

Remmert, M., et al. HHblits: lightning-fast iterative protein sequence searching by HMM-HMM alignment. Nature methods 2012;9(2):173.

Rohl, C.A., et al. Protein structure prediction using Rosetta. Methods in enzymology 2004;383:66-93.

Rost, B. Protein secondary structure prediction continues to rise. Journal of structural biology 2001;134(2-3):204-218.

Roy, A., Kucukural, A. and Zhang, Y. I-TASSER: a unified platform for automated protein structure and function prediction. Nature protocols 2010;5(4):725-738.

Schmidler, S.C., Liu, J.S. and Brutlag, D.L. Bayesian segmentation of protein secondary structure. Journal of computational biology 2000;7(1-2):233-248.

Spencer, M., Eickholt, J. and Cheng, J. A deep learning network approach to ab initio protein secondary structure prediction. IEEE/ACM transactions on computational biology and bioinformatics 2015;12(1):103-112.

Srivastava, N., et al. Dropout: a simple way to prevent neural networks from overfitting. Journal of Machine Learning Research 2014;15(1):1929-1958.

Stolorz, P., Lapedes, A. and Xia, Y. Predicting protein secondary structure using neural net and statistical methods. Journal of Molecular Biology 1992;225(2):363377 .

Szegedy, C., et al. Going deeper with convolutions. In, Proceedings of the IEEE conference on computer vision and pattern recognition. 2015. p. 1-9.

Torrisi, M., Kaleel, M. and Pollastri, G. Porter 5: fast, state-of-the-art ab initio prediction of protein secondary structure in 3 and 8 classes. bioRxiv 2018:289033.

Uziela, K., et al. ProQ3: Improved model quality assessments using Rosetta energy terms. Scientific reports 2016;6:33509.

Wang, G. and Dunbrack Jr, R.L. PISCES: a protein sequence culling server. Bioinformatics 2003;19(12):1589-1591.

Wang, S., et al. Protein secondary structure prediction using deep convolutional neural fields. Scientific reports 2016;6.

Wang, S., et al. Accurate De Novo Prediction of Protein Contact Map by Ultra-Deep Learning Model. PLOS Computational Biology 2017;13(1):e1005324.

Wang, Z., Eickholt, J. and Cheng, J. MULTICOM: a multi-level combination approach to protein structure prediction and its assessments in CASP8. Bioinformatics 2010;26(7):882-888.

Webb, B. and Sali, A. Protein structure modeling with MODELLER. Protein Structure Prediction 2014:1-15.

Yan, R., et al. A comparative assessment and analysis of 20 representative sequence alignment methods for protein structure prediction. Scientific reports 2013;3:2619.

Yang, Y., et al. Sixty-five years of the long march in protein secondary structure prediction: the final stretch? Briefings in bioinformatics 2016:bbw129.

Zemla, A., et al. A modified definition of Sov, a segment - based measure for protein secondary structure prediction assessment. Proteins: Structure, Function, and Bioinformatics 1999;34(2):220-223. 